\title{
The Orbit Structure of Cantor Minimal $\mathbb{Z}^{2}$-Systems
}

\author{
Thierry Giordano ${ }^{1} *$, Ian F. Putnam ${ }^{2} \dagger$, and Christian F. Skau ${ }^{3}$ \\ 1 Department of Mathematics and Statistics, University of Ottawa, 585 King \\ Edward Ave., Ottawa, Ontario, Canada K1N 6N5, giordano@uottawa.ca. \\ 2 Department of Mathematics and Statistics, University of Victoria, Victoria, \\ B.C., Canada V8W 3P4, putnam@math.uvic.ca. \\ 3 Norwegian University of Science and Technology (NTNU), N-7491 Trondheim, \\ Norway, csk@math.ntnu.no.
}

\section{Introduction.}

In 1959, H. Dye ([D1]) introduced the notion of orbit equivalence and proved that any two ergodic finite measure preserving transformations on a Lebesgue space are orbit equivalent. In [D2], he had also conjectured that an arbitrary ergodic action of a discrete amenable group is orbit equivalent to a $\mathbb{Z}$-action. This conjecture was proved by Ornstein and Weiss in [OW]. The most general case was proved by Connes, Feldman and Weiss ([CFW]) by establishing that an amenable non-singular countable equivalence relation $\mathcal{R}$ can be generated by a single transformation, or equivalently, is hyperfinite, i.e., $\mathcal{R}$ is up to a null set, a countable increasing union of finite equivalence relations.

For the Borel case, Weiss ([W]) proved that actions of $\mathbb{Z}^{n}$ are (orbit equivalent to) hyperfinite Borel equivalence relations, whose classification was obtained by Dougherty, Jackson and Kechris ([DJK]). It is not yet known if an arbitrary Borel action of a discrete amenable group is orbit equivalent to a $\mathbb{Z}$-action.

Our main interest in this report is the case of a free minimal continuous action $\varphi$ of $\mathbb{Z}^{2}$ on a Cantor set (i.e., a compact totally disconnected metric space with no isolated points). However, let us begin with a more general group action and consider a free action $\varphi$ of a countable discrete group on a compact metric space $X$ (i.e., for every $g \in G, \varphi(g) \in \operatorname{Homeo}(X)$, and $\varphi(g) x=x$ for some $x \in X$ if and only if $g=i d)$. Recall that the action $\varphi$ is minimal if the $\varphi$-orbit of every point of $X$ is dense in $X$.

\footnotetext{
* Research supported in part by operating grants from NSERC (Canada).

$\dagger$ Research supported in part by operating grants from NSERC (Canada).

$\ddagger$ Research supported in part by the Norwegian Research Council for Science and Humanities.
} 
Given two free group actions $(X, G, \varphi)$ and $(Y, H, \psi)$, an isomorphism between them is a homeomorphism $h: X \rightarrow Y$ and a group isomorphism $\alpha: G \rightarrow H$ such that, for all $g \in G$, we have

$$
h \circ \varphi(g)=\psi(\alpha(g)) \circ h .
$$

Recall from [GPS2] that, given two free group actions $(X, G, \varphi)$ and $(Y, H, \psi)$, an orbit equivalence between them is a homeomorphism $h: X \rightarrow Y$ such that, for every $x \in X$, we have

$$
h\left(\mathcal{O}_{\varphi}(x)\right)=\mathcal{O}_{\psi}(h(x)),
$$

where $\mathcal{O}_{\varphi}(x)$ denotes the orbit of the point $x \in X$ under the action of $\varphi$. It is clear from the definitions that every isomorphism is also an orbit equivalence.

For connected spaces, using a result of Sierpinski (see $[\mathrm{K}]$, Thm 6, Ch $\mathrm{V}, 47$, III), any orbit equivalence is also an isomorphism. Therefore, we will consider only spaces which are totally disconnected.

\section{2 Étale equivalence relations}

Let $X$ be a compact metric space and $G$ be a countable group with the discrete topology. If $\varphi$ is a free continuous action of $G$ on $X$, let $\mathcal{R}_{\varphi}$ denote the equivalence relation given by

$$
\mathcal{R}_{\varphi}=\{(x, \varphi(g) x) ; x \in X, g \in G\} .
$$

With the product topology, $X \times G$ is a $\sigma$-compact, locally compact space; then using the bijection from $X \times G$ to $\mathcal{R}_{\varphi}$ given by $(x, g) \mapsto(x, \varphi(g) x)$, the equivalence relation $\mathcal{R}_{\varphi}$ becomes a topological groupoid. If $r$ and $s$ (for range and source) denote the two canonical projections from $\mathcal{R}_{\varphi}$ to $X$ :

$$
s(x, \varphi(g) x)=\varphi(g) x \quad \text { and } \quad r(x, \varphi(g) x)=x,
$$

then $r$ and $s$ are local homeomorphisms. Moreover as $G$ is countable, each $\mathcal{R}_{\varphi}$ is a countable equivalence relation, i.e. each equivalence class $[x]_{\mathcal{R}_{\varphi}}=\{y \in$ $\left.X \mid(x, y) \in \mathcal{R}_{\varphi}\right\}$ is countable for each $x \in X$. Then $\mathcal{R}_{\varphi}$ is the motivating example of an étale equivalence relation, whose precise definition is as follows:

Definition 1. The locally compact groupoid $(\mathcal{R}, \mathcal{T})$, where $\mathcal{R}$ is a countable equivalence relation on a compact metric space $X$, is étale if the maps $r, s$ : $\mathcal{R} \rightarrow X$ are local homeomorphisms, i.e. for every $(x, y) \in \mathcal{R}$ there exists an open neighborhood $U \in \mathcal{T}$ of $(x, y)$ so that $r(U)$ and $s(U)$ are open in $X$ and $r: U \rightarrow r(U)$ and $s: U \rightarrow s(U)$ are homeomorphisms. If $X$ is zerodimensional, we may clearly choose $U$ to be a clopen set.

We will call $(\mathcal{R}, \mathcal{T})$ an étale equivalence relation on $X$. 
Remark 2.

a) This definition is equivalent to the various definitions of an étale (or rdiscrete) locally compact groupoid (applied in our setting) that can be found in the literature (see for example [Pa], $[\mathrm{R}]$ ).

b) If $\mathcal{R}$ is an étale equivalence relation, then its equivalence classes are countable. By definition, $\mathcal{R}$ can be written as a union of graphs of local homeomorphisms of the form $s \circ r^{-1}$.

c) The topology $\mathcal{T}$ on $\mathcal{R}$ is rarely the relative topology from $\mathcal{R} \subset X \times X$. Indeed if $\mathcal{R}$ is étale and has an infinite equivalence class, then $\mathcal{T}$ is not the relative topology of $X \times X$.

d) A countable equivalence relation $\mathcal{R}$ on $X$ may be given distinct nonisomorphic topologies $\mathcal{T}_{1}$ and $\mathcal{T}_{2}$ so that $\left(\mathcal{R}, \mathcal{T}_{1}\right)$ and $\left(\mathcal{R}, \mathcal{T}_{2}\right)$ are étale equivalence relations. This contrasts with the situation in the countable (standard) Borel equivalence relation setting, where the Borel structure is uniquely determined by $\mathcal{R} \subset X \times X$.

Generalizing the statement and the proof of Theorem 1 of $[\mathrm{FM}]$, we have:

Proposition 3. Let $(\mathcal{R}, \mathcal{T})$ be an étale equivalence relation on the zerodimensional space $X$. There exists a countable group $G$ of homeomorphisms of $X$ so that $\mathcal{R}=\mathcal{R}_{G}$, where $\mathcal{R}_{G}=\{(x, g x) ; x \in X, g \in G\}$.

Remark 4. In [HM], Hjorth and Molberg have recently shown that the group $G$ in Proposition 3 cannot always be chosen acting freely.

There are two natural notions of equivalence between étale equivalence relations:

Definition 5. (Isomorphism and orbit equivalence) Let $\left(\mathcal{R}_{1}, \mathcal{T}_{1}\right)$ and $\left(\mathcal{R}_{2}, \mathcal{T}_{2}\right)$ be two étale equivalence relations on $X_{1}$ and $X_{2}$ respectively.

1. $\left(\mathcal{R}_{1}, \mathcal{T}_{1}\right)$ and $\left(\mathcal{R}_{2}, \mathcal{T}_{2}\right)$ are orbit equivalent if there exists a homeomorphism $F: X_{1} \rightarrow X_{2}$ so that

$$
(x, y) \in \mathcal{R}_{1} \Longleftrightarrow(F(x), F(y)) \in \mathcal{R}_{2} .
$$

We call such a map $F$ an orbit map.

2. $\left(\mathcal{R}_{1}, \mathcal{T}_{1}\right)$ and $\left(\mathcal{R}_{2}, \mathcal{T}_{2}\right)$ are isomorphic if there is an orbit map $F: X_{1} \rightarrow X_{2}$ so that $F \times F:\left(\mathcal{R}_{1}, \mathcal{T}_{1}\right) \rightarrow\left(\mathcal{R}_{2}, \mathcal{T}_{2}\right)$ is a homeomorphism.

Observe that $\left(\mathcal{R}_{1}, \mathcal{T}_{1}\right)$ is orbit equivalent to $\left(\mathcal{R}_{2}, \mathcal{T}_{2}\right)$, via the orbit map $F$ if and only if $F\left([x]_{\mathcal{R}_{1}}\right)=[F(x)]_{\mathcal{R}_{2}}$ for each $x \in X_{1}$. So $F$ maps equivalence classes into equivalence classes.

There is a notion, introduced by J. Renault ([R1]), of an invariant probability measure for an étale equivalence relation $\mathcal{R} \subset X \times X$. A measure $\mu$ on $X$ is $\mathcal{R}$-invariant if $\mu(r(U))=\mu(s(U))$, for every open set $U \subset \mathcal{R}$ such that $r: U \rightarrow r(U)$ and $s: U \rightarrow s(U)$ are homeomorphisms. We will denote by $M(X, \mathcal{R})$ the compact convex cone of $\mathcal{R}$-invariant probability measures on $X$. If $F: X_{1} \rightarrow X_{2}$ is an orbit equivalence between two étale equivalence relations, then $F$ induces a bijection between the two sets of invariant probability measures $M\left(X_{1}, \mathcal{R}_{1}\right)$ and $M\left(X_{2}, \mathcal{R}_{2}\right)$. 


\section{Invariants for Cantor étale equivalence relation}

To an étale equivalence relation $\mathcal{R}$ on the Cantor set $X$ we associate two ordered groups which are invariants of isomorphism and orbit equivalence of $\mathcal{R}$.

By an ordered group, we mean a countable abelian group $G$ with a subset $G^{+}$, called the positive cone, such that
(i) $G^{+}+G^{+} \subset G^{+}$,
(ii) $G^{+}-G^{+}=G$,
(iii) $G^{+} \cap\left(-G^{+}\right)=\{0\}$.

By an order unit for $\left(G, G^{+}\right)$, we mean an element $u \in G^{+}$such that for every $a \in G^{+}, n u-a \in G^{+}$, for some $n \geq 1$.

Let $C(X, \mathbb{Z})$ be the abelian group of continuous functions with values in $\mathbb{Z}$. We denote by $B(X, \mathcal{R})$ the (coboundary) subgroup of $C(X, \mathbb{Z})$ generated by the functions $\chi_{r(U)}-\chi_{s(U)}$, where $U$ is a clopen subset of $\mathcal{R}$ on which $r$ and $s$ are local homeomorphisms.

We define $B_{m}(X, \mathcal{R})$ to be the subgroup of $C(X, \mathbb{Z})$ of all functions $f$ such that $\int_{X} f d \mu=0$, for all $\mu \in M(X, \mathcal{R})$. Note that if $M(X, \mathcal{R})=\emptyset$, then $B_{m}(X, \mathcal{R})=C(X, \mathbb{Z})$.

Definition 6. Let $\mathcal{R}$ be an étale equivalence relation on the Cantor set $X$. We denote by

i) $D(X, \mathcal{R})=C(X, \mathbb{Z}) / B(X, \mathcal{R})$ the ordered group whose positive cone and order unit u are

$$
D(X, \mathcal{R})^{+}=\{[f] ; f \in C(X, \mathbb{Z}), f \geq 0\} \quad \text { and } \quad u=[1] .
$$

ii) $D_{m}(X, \mathcal{R})=C(X, \mathbb{Z}) / B_{m}(X, \mathcal{R})$ the ordered group whose positive cone and order unit $u$ are

$$
D_{m}(X, \mathcal{R})^{+}=\{[f] ; f \in C(X, \mathbb{Z}), f \geq 0\} \quad \text { and } \quad u=[1] .
$$

Remark \%.

i) $B(X, \mathcal{R})$ is a subset of $B_{m}(X, \mathcal{R})$ and $D_{m}(X, \mathcal{R})$ is a quotient of $D(X, \mathcal{R})$. ii) If $\mathcal{R}_{\varphi}$ denotes the étale equivalence relation induced by a minimal homeomorphism $\varphi$ of the Cantor set $X$, we have:

- By $[\mathrm{P}]$, Thm 4.1 and [HPS], Cor. 6.3 , the triple $\left(D\left(X, \mathcal{R}_{\varphi}\right), D\left(X, \mathcal{R}_{\varphi}\right)^{+},[1]\right)$ is a simple, acyclic dimension group with (canonical) order unit. Moreover any simple, acyclic dimension group $\left(G, G^{+}, u\right)$ where $u$ is a distinguished order unit, can be realized as $\left(D\left(X, \mathcal{R}_{\varphi}\right), D\left(X, \mathcal{R}_{\varphi}\right)^{+},[1]\right)$ for a Cantor minimal system $(X, \varphi)$.

- $B_{m}\left(X, \mathcal{R}_{\varphi}\right) / B\left(X, \mathcal{R}_{\varphi}\right)$ is equal to the infinitesimal subgroup $\operatorname{Inf}\left(D\left(X, \mathcal{R}_{\varphi}\right)\right)$ of $D\left(X, \mathcal{R}_{\varphi}\right)$ and $D\left(X, \mathcal{R}_{\varphi}\right) / \operatorname{Inf}\left(D\left(X, \mathcal{R}_{\varphi}\right)\right)$ is naturally isomorphic to $D_{m}\left(X, \mathcal{R}_{\varphi}\right)$.

It is then easy to check that: 
Proposition 8. If $F: X_{1} \rightarrow X_{2}$ is an orbit map between two étale equivalence relations $\left(X_{1}, \mathcal{R}_{1}, \mathcal{T}_{1}\right)$ and $\left(X_{2}, \mathcal{R}_{2}, \mathcal{T}_{2}\right)$, then it induces an order isomorphism preserving the order units from $D_{m}\left(X_{1}, \mathcal{R}_{1}\right)$ to $D_{m}\left(X_{2}, \mathcal{R}_{2}\right)$.

Moreover if $F$ implements an isomorphism between $\left(X_{1}, \mathcal{R}_{1}, \mathcal{T}_{1}\right)$ and $\left(X_{2}, \mathcal{R}_{2}, \mathcal{T}_{2}\right)$, then it induces an order isomorphism preserving the order units from $D\left(X_{1}, \mathcal{R}_{1}\right)$ to $D\left(X_{2}, \mathcal{R}_{2}\right)$.

\section{AF-equivalence relations}

The AF equivalence relations ([R1], [GPS2]) form one of the most important classes of étale equivalence relations. The terminology $\mathrm{AF}$ comes from $\mathrm{C}^{*}$ algebra theory and means approximately finite.

Definition 9. An étale equivalence relation $\mathcal{R}$ on $X$ is an AF-relation if $X$ is a totally disconnected compact metrizable space and if there are

$$
\mathcal{R}_{1} \subset \mathcal{R}_{2} \subset \cdots
$$

such that $\cup_{n} \mathcal{R}_{n}=\mathcal{R}$ and $\mathcal{R}_{n} \subset \mathcal{R}$ is a compact open subequivalence relation, for each $n \geq 1$.

Before giving examples of AF-equivalence relations, let us note that:

Proposition 10. ([GPS2], Thm 3.8). Let $\varphi$ be a free action of a countable group $G$ on a totally disconnected compact metric space $X$. The relation $\mathcal{R}_{\varphi}$ is an AF-equivalence relation if and only if the group $G$ is locally finite.

Let us describe the fundamental example of an AF-equivalence relation. We begin with a Bratteli diagram (see [HPS], [Ef]). It is a locally finite, infinite directed graph which consists of a vertex set $V$ and an edge set $E$ written as a countable disjoint union of non-empty finite sets:

$$
V=V_{0} \cup V_{1} \cup V_{2} \cup \cdots \text { and } \quad E=E_{1} \cup E_{2} \cup E_{3} \cup \cdots
$$

Each edge $e$ in $E_{n}$ has a source $i(e)$ in $V_{n-1}$, and a range $f(e)$ in $V_{n}$. For simplicity we assume that $V_{0}$ consists of a single vertex and for every other vertex $v, f^{-1}\{v\}$ and $i^{-1}\{v\}$ are non-empty.

The space $X=X_{(V, E)}=\left\{e=\left(e_{1}, e_{2}, \cdots\right) ; e_{n} \in E_{n}, i\left(e_{n+1}\right)=\right.$ $f\left(e_{n}\right)$ for $\left.n \geq 1\right\}$ is the space of infinite paths in the diagram. It is given the relative topology of the product space $\prod_{n} E_{n}$ and is therefore compact metrizable and zero dimensional. For each $N \geq 0$, let

$$
\mathcal{R}_{N}=\left\{(e, f) \in X \times X ; e_{n}=f_{n} \text { for all } n>N\right\} .
$$

With the relative topology of the product $X \times X$, then $\mathcal{R}_{N}$ is a compact étale equivalence relation (hence each equivalence class is finite), and $\mathcal{R}_{N}$ is an open subset of $\mathcal{R}_{N+1}$, for all $N \geq 1$. 
Let $\mathcal{R}=\bigcup_{N=0}^{\infty} \mathcal{R}_{N}$, and give $\mathcal{R}$ the inductive limit topology. This means that a sequence $\left\{\left(x_{n}, y_{n}\right)\right\}$ in $\mathcal{R}$ converges to $(x, y)$ in $\mathcal{R}$ if and only if $\left\{x_{n}\right\}$ converges to $x,\left\{y_{n}\right\}$ converges to $y$ (in $X$ ) and, for some $N,\left(x_{n}, y_{n}\right)$ is in $R_{N}$ for all but finitely many $n$. Then $\mathcal{R}$ is easily seen to be an étale equivalence relation which will be denoted by $A F(V, E)$.

We observe that if $\left(V^{\prime}, E^{\prime}\right)$ is a telescope of $(V, E)$, i.e. $\left(V^{\prime}, E^{\prime}\right)$ is obtained from $(V, E)$ by telescoping $(V, E)$ to certain levels $0<n_{1}<n_{2}<n_{3}<\cdots$, then $A F(V, E)$ is isomorphic to $A F\left(V^{\prime}, E^{\prime}\right)$. In fact, there is a natural homeomorphism $\alpha: X_{(V, E)} \rightarrow X_{\left(V^{\prime}, E^{\prime}\right)}$, and $\alpha$ clearly implements the isomorphism, according to the description we have given of convergence in $A F(V, E)$, respectively $A F\left(V^{\prime}, E^{\prime}\right)$.

The Bratteli diagram $(V, E)$ is simple if for each $n$ there is an $m>n$ so that by telescoping the diagram between levels $n$ and $m$, every vertex $v$ in $V_{n}$ is connected to every vertex $w$ in $V_{m}$. It is a simple observation that $(V, E)$ is simple if and only if every $A F(V, E)$-equivalence class is dense in $X_{(V, E)}$.

The above example is in fact the general case. Indeed, we have:

Theorem 11. Let $\mathcal{R}$ be an AF-relation on a totally disconnected compact metrizable space $X$. Then there exists a Bratteli diagram $(V, E)$ such that $\mathcal{R}$ is isomorphic to the $A F$-equivalence relation $A F(V, E)$ associated to $(V, E)$.

Furthermore, $(V, E)$ is simple if and only if $\mathcal{R}$ is minimal (i.e. every $\mathcal{R}$ equivalence class is dense).

\section{The classification of AF-equivalence relations}

For AF-equivalence relations the invariants introduced in Definition 3.1 have not only a well-known structure, but they also form complete sets of invariants of AF-equivalence relations up to isomorphism and in the minimal case up to orbit equivalence. Indeed we have:

Theorem 12. (see $[H P S]$ ) For a Bratteli diagram $(V, E)$ and the associated $A F$-equivalence relation $A F(V, E)$, the group $D\left(X_{(V, E)}, A F(V, E)\right)$ is the dimension group of the Bratteli diagram $(V, E)$. It is simple if and only if $A F(V, E)$ is minimal.

Approximately finite dimensional $\mathrm{C}^{*}$-algebras were classified in 1976 by G. Elliott. Building on this result, Krieger proved in $[\mathrm{Kr}]$ the following:

Theorem 13. For AF-equivalence relations $(X, \mathcal{R})$, the triple formed by the ordered group $\left(D(X, \mathcal{R}), D(X, \mathcal{R})^{+}\right)$and the order unit [1] is a complete invariant for isomorphism.

For minimal AF-relations, we then can get:

Theorem 14. For AF-equivalence relations $(X, \mathcal{R})$, the triple formed by the dimension group $\left(D_{m}(X, \mathcal{R}), D_{m}(X, \mathcal{R})^{+}\right)$and the order unit [1] is a complete invariant for orbit equivalence. 
Even if Theorem 5.2 appears in [GPS1] as a corollary of the classification up to orbit equivalence of minimal $\mathbb{Z}$-actions, a direct proof can be given and it is in fact more logical to do so. Note that the range of the invariant for orbit equivalence of AF-equivalence relations is the class of all simple, acyclic dimension groups whose the infinitesimal subgroup is trivial.

\section{The Strategy for Orbit Equivalence Results}

Let $G$ be $\mathbb{Z}$ or $\mathbb{Z}^{2}$ and $\varphi$ be a minimal, free $G$-action on the Cantor set $X$. As the classification of AF-equivalence relations up to orbit equivalence is known, it is sufficient to show that such an action is affable (i.e., orbit equivalent to an AF-equivalence relation). This will be achieved with the following two steps:

\section{(1)}

If $\varphi$ and $G$ are as above, construct a minimal AF-subequivalence relation $\mathcal{R}$ of $\mathcal{R}_{\varphi}$, two closed "small" subsets $Y_{0}$ and $Y_{1}$ of $X$, and a homeomorphism $\alpha: Y_{0} \rightarrow Y_{1}$ such that the equivalence relation $\mathcal{R} \vee$ Graph $(\alpha)$, generated by $\mathcal{R}$ and the graph of $\alpha$ is equal to $\mathcal{R}_{\varphi}$.

Prove then that $\mathcal{R} \vee \operatorname{Graph}(\alpha)$ is orbit equivalent to $\mathcal{R}$. The second step means that a minimal AF-relation $\mathcal{R}$ can be enlarged "slightly" and stays $\mathrm{AF}$, more precisely still be orbit equivalent to $\mathcal{R}$. We will present the precise statement in the next section.

The first step depends on the group $G$. We have a complete answer for $G=\mathbb{Z}$ and up to now only a partial one for $\mathbb{Z}^{2}$.

Remark 15. For $G=\mathbb{Z}$, this strategy was used by Dye in the measurable case using repetitively the Rohlin lemma to get the first step, with the small subsets $Y_{0}$ and $Y_{1}$ having measure zero. This can be extended to include amenable groups. The second step is then not necessary.

For Borel actions of $\mathbb{Z}^{n}$, Weiss ([W]) used also the same strategy. Contrary to the (finite invariant measure) measurable case, AF-relations are not unique. They were classified by Dougherty, Jackson and Kechris ([DJK]) and their complete invariant up to orbit equivalence is the cardinality of the set of their finite invariant ergodic measures.

\section{The Absorption Theorem}

The second step of our strategy for orbit equivalence results will be accomplished with Theorem 7.1. This result states precise sufficient conditions under 
which a minimal AF-relation can be enlarged and stay orbit equivalent to itself. For an étale equivalence relation $\mathcal{R}$ on $X$, let us recall some terminology: if $Y$ is a closed subset of $X$, we say that:

1)

$Y$ is $\mathcal{R}$-étale if $\left.\mathcal{R}\right|_{Y}(=\mathcal{R} \cap(Y \times Y))$, with the relative topology, is an étale equivalence relation on $Y$.

2)

$Y$ is a thin subset if $\mu(Y)=0$ for every finite $\mathcal{R}$-invariant measure $\mu$. 
Theorem 16. ([GPS2], Thm 4.18). Let $\mathcal{R}$ be a minimal AF-equivalence relation on the Cantor set $X$ and let $Y_{0}, Y_{1}$ be two closed $\mathcal{R}$-étale and thin subsets of $X$. Suppose $\mathcal{R} \cap\left(Y_{0} \times Y_{1}\right)=\emptyset$ ( and so in particular $\left.Y_{0} \times Y_{1}=\emptyset\right)$, and let $\alpha: Y_{0} \rightarrow Y_{1}$ be a homeomorphism such that $\alpha \times \alpha:\left.\left.\mathcal{R}\right|_{Y_{0}} \rightarrow \mathcal{R}\right|_{Y_{1}}$ is an isomorphism.

Then the equivalence relation on $X$

$$
\mathcal{R} \vee\left\{(y, \alpha(y)) ; y \in Y_{0}\right\}
$$

generated by $\mathcal{R}$ and Graph $(\alpha)$ is orbit equivalent to $\mathcal{R}$ and therefore is affable.

\section{Classification up to orbit equivalence of minimal $\mathbb{Z}$-actions}

Let $\varphi$ be a Cantor minimal system (i.e. a minimal action of $\mathbb{Z}$ on the Cantor set). The first step of the strategy outlined in section 6 , namely to show that $\mathcal{R}_{\varphi}$ is affable is based on the following construction, that we sketch now.

Let $\left(U_{n}\right)_{n \geq 1}$ be a decreasing sequence of clopen subsets of $X$, whose intersection is a single point $y$. For $n \geq 1$, let $\mathcal{R}_{n}$ denote the equivalence relation on $X$ generated by $\left\{(x, \varphi(x)) \mid x \in X \backslash U_{n}\right\}$. As $\varphi$ is minimal and as the first return map of $\varphi$ on $U_{n}$ is continuous, we have that $\mathcal{R}_{n}$ is compact and open. As $\left(U_{n}\right)_{n \geq 1}$ forms a decreasing sequence of clopen sets, the sequence of the equivalence relations $\left(\mathcal{R}_{n}\right)_{n \geq 1}$ is increasing and their union $\mathcal{R}_{y}$ is an AF-relation. Every $\mathcal{R}_{y}$-class is also a $\varphi$-orbit, except for the orbit of the point $y$ and $\mathcal{R}_{\varphi}=\mathcal{R}_{y} \vee\{(y, \varphi(y))\}$. With $Y_{0}=\{y\}, Y_{1}=\{\varphi(y)\}$ and $\alpha=\varphi$, we can apply Theorem 7.1 and we get:

Theorem 17. ([GPS1]). Let $\varphi$ be a Cantor minimal system. Then the equivalence relation $\mathcal{R}_{\varphi}$ is orbit equivalent to an $A F$-relation.

As a consequence of this result and of Theorem 5.2, we have:

Theorem 18. Two Cantor minimal systems $\varphi$ and $\psi$ are orbit equivalent if and only if $\left(D_{m}\left(X, \mathcal{R}_{\varphi}\right), D_{m}\left(X, \mathcal{R}_{\varphi}\right)^{+},[1]\right)$ and $\left(D_{m}\left(X, \mathcal{R}_{\psi}\right), D_{m}\left(X, \mathcal{R}_{\psi}\right)^{+},[1]\right)$ are (order) isomorphic.

Remark 19. a) Let $\varphi$ be a Cantor minimal system and $C^{*}(X, \varphi)$ be the associated $\mathrm{C}^{*}$-crossed product. If $\mu$ is any $\varphi$-invariant probability measure on $X$, recall that $C^{*}(X, \varphi)$ can be realized as the $\mathrm{C}^{*}$-subalgebra of the bounded linear operator algebra $\mathcal{B}\left(L^{2}(X, \mu)\right)$ generated by $C(X)$ acting as multiplication operators and the unitary operator $u=u_{\varphi}$, defined by

$$
u \xi(x)=\xi\left(\varphi^{-1} x\right), \text { for } x \in X \text { and } \xi \in L^{2}(X, \mu) .
$$

If $Y$ is a non-empty closed subset of $X$, let $A_{Y}^{\varphi}$ be the $\mathrm{C}^{*}$-subalgebra of $C^{*}(X, \varphi)$ generated by $C(X)$ and $u C_{0}(X \backslash Y)$, where $C_{0}(X \backslash Y)$ denotes the continuous functions vanishing on $Y$.

Let $\left(U_{n}\right)_{n \geq 1}$ be a decreasing sequence of clopen subsets of $X$, whose intersection is a single point $y$. In $[\mathrm{P}]$, I. Putnam proved that 
$A_{y}^{\varphi}$ is an AF algebra which is the closure of the increasing union of the finite dimensional algebras $A_{U_{n}}^{\varphi}$, for $n \geq 1$

the inclusion map $i: A_{y}^{\varphi} \rightarrow C^{*}(X, \varphi)$ induces an isomorphism $i_{*}: K_{0}\left(A_{y}^{\varphi}\right) \rightarrow$ $K_{0}\left(C^{*}(X, \varphi)\right)$ of ordered groups preserving the distinguished order units (the class of the identity operator).

$K_{0}\left(C^{*}(X, \varphi)\right)$ is order isomorphic to $D\left(X, \mathcal{R}_{\varphi}\right)$ by a map preserving the order units.

Let $C^{*}(\mathcal{S})$ denote the reduced $\mathrm{C}^{*}$-algebra associated to an étale equivalence relation $\mathcal{S}$ (see $[\mathrm{Pa}]$ and $[\mathrm{R}]$ for example). If for $n \geq 1$, as introduced above, $\mathcal{R}_{n}$ denotes the equivalence relation generated by $\{(x, \varphi(x)) \mid x \in$ $\left.X \backslash U_{n}\right\}$ and $\mathcal{R}_{y}$ their union, then $C^{*}\left(\mathcal{R}_{n}\right)$ is isomorphic to $A_{U_{n}}^{\varphi}$ and $A_{y}^{\varphi}$ to $C^{*}\left(\mathcal{R}_{y}\right)$.

If $\varphi$ and $\psi$ are two orbit-equivalent minimal homeomorphisms acting on the Cantor set $X$ and if $F \in \operatorname{Homeo}(X)$ is an orbit map between them, recall that the orbit cocycles $m$ an $n$ associated to $F$ are the integer-valued functions on $X$ defined for $x \in X$, by

$$
F \circ \varphi(x)=\psi^{n(x)} \circ F(x) \text { and } F \circ \varphi^{m(x)}(x)=\psi \circ F(x) .
$$

By a theorem of M. Boyle (see [GPS1], Thm 1.4), if one of the orbit cocycles is continuous, then $\varphi$ and $\psi$ are flip-conjugate (i.e., $\varphi$ is conjugate to either $\psi$ or $\left.\psi^{-1}\right)$.

Definition 20. Let $\varphi$ and $\psi$ be two orbit-equivalent minimal homeomorphisms acting on the Cantor set $X$. Then $\varphi$ and $\psi$ are strong orbit equivalent if there exists an orbit map $F$ so that the associated orbit cocycles $m, n: X \rightarrow \mathbb{Z}$ each have at most one point of discontinuity.

Let us finish this section on the classification of Cantor minimal systems by stating the following two results:

Theorem 21. Let $\varphi$ and $\psi$ be two minimal homeomorphisms acting on the Cantor set $X$. Then the two étale equivalence relations $\mathcal{R}_{\varphi}$ and $\mathcal{R}_{\psi}$ are isomorphic if and only if $\varphi$ and $\psi$ are flip-conjugate.

Theorem 22. Let $\varphi$ and $\psi$ be two minimal homeomorphisms acting on the Cantor set $X$. For any two points $y_{1}$ and $y_{2}$ of $X$, the AF-equivalence relations $\mathcal{R}_{\varphi, y_{1}}$ and $\mathcal{R}_{\psi, y_{2}}$ are isomorphic if and only if $\varphi$ and $\psi$ are strongly orbit equivalent. 
For the first result, if $\varphi$ and $\psi$ are flip-conjugate, then $\mathcal{R}_{\varphi}$ and $\mathcal{R}_{\psi}$ are clearly isomorphic. Conversely, if $F$ is an orbit map such that $F \times F$ is a homeomorphism from $\mathcal{R}_{\varphi}$ to $\mathcal{R}_{\psi}$, then it follows that the orbit cocycles are bounded and by M. Boyle's theorem $\varphi$ and $\psi$ are flip-conjugate.

The second one follows from [GPS2], Lemma 4.13 and Corollary 1.3, in combination with Theorem 2.1 of [GPS1].

\section{Classification up to orbit equivalence of minimal $\mathbb{Z}^{2}$-actions}

Let $\varphi$ be a minimal free action of $\mathbb{Z}^{2}$ on the Cantor set. To fulfill the second step of the strategy outlined in section 6 , we use cocycles for the action to create the AF-relation and its extension. The drawback of this method is that it needs to assume the existence of sufficiently many cocycles with conditions of positivity and smallness we will define below. Results about the existence are still partial, although they do exist for several examples of interest.

In $[\mathrm{F}]$, Forrest (see also $[\mathrm{Ph}]$ for another treatment) produced large AFsubrelations of the orbit relation $\mathcal{R}_{\varphi}$. Such subrelations also appear implicitly in works of Bellissard, Benedetti and Gambaudo [BBG] and also in Benedetti and Gambaudo [BG]. But their methods do not keep track of the difference between the AF-subrelation and $\mathcal{R}_{\varphi}$ and therefore do not allow the use of the absorption theorem.

\subsection{Cocycles and Positive Cocycles}

Before stating our main results, we need to recall some basic notions about cocycles whose basic references are [FM, R].

Definition 23. Let $\varphi$ be a free action of $\mathbb{Z}^{2}$ on a compact space $X$.

$A \mathbb{Z}$-valued one-cocycle for $\varphi$ is a continuous function $\theta: X \times \mathbb{Z}^{2} \rightarrow \mathbb{Z}$ such that, for all $x \in X$ and $m, n \in \mathbb{Z}^{2}$, we have

$$
\theta(x, m+n)=\theta(x, m)+\theta\left(\varphi^{m}(x), n\right) .
$$

If $f \in C(X, \mathbb{Z})$, then the function $b f(x, n)=f\left(\varphi^{n}(x)\right)-f(x)$ is called a coboundary.

The set $Z^{1}(X, \varphi)$ of all cocycles forms a group under addition, the set $B^{1}(X, \varphi)$ of all coboundaries a subgroup and we denote by $H^{1}(X, \varphi)=$ $Z^{1}(X, \varphi) / B^{1}(X, \varphi)$ the first cohomology group. Using the equivalence relation induced by $\varphi$ on $X$, cocycles and coboundaries can also be viewed as continuous homomorphisms from $\mathcal{R}_{\varphi}$ to $\mathbb{Z}$. Therefore if $\theta$ is a cocycle, its kernel $\operatorname{ker}(\theta)=\left\{(x, y) \in \mathcal{R}_{\varphi} \mid \theta(x, y)=0\right\}$ is a closed subequivalence of $\mathcal{R}_{\varphi}$.

We introduce now the notion of strict positivity for cocycles.

Definition 24. Let $\varphi$ be a free action of $\mathbb{Z}^{2}$ on a compact space $X$ and let $C$ be a subset of $\mathbb{Z}^{2}$. If $\theta$ is a cocycle, then it is 
1.

positive with respect to $C$ if $\theta(X \times C) \geq 0$.

2.

proper with respect to $C$ if the map $\theta: X \times C \rightarrow \mathbb{Z}$ is proper (i.e., the pre-image of any finite set is compact).

3.

strictly positive with respect to $C$ if it is proper and positive with respect to $C$.

Condition (2) of this definition is the key property we use to produce compact open subequivalence of $\mathcal{R}_{\varphi}$. Indeed we have:

Proposition 25. ([GPS3], Prop. 5.12) Let $(X, \varphi)$ be as in 9.2, $\xi$ and $\eta$ be cocycles for $(X, \varphi)$ and let $C, C^{\prime} \subset \mathbb{Z}^{2}$. If $\xi$ is proper on $C$ and $\eta$ is proper on $C^{\prime}$ and

$$
C \cup(-C) \cup C^{\prime} \cup\left(-C^{\prime}\right)=\mathbb{Z}^{2},
$$

then $\operatorname{ker}(\xi) \cap \operatorname{ker}(\eta)=\left\{(x, y) \in \mathcal{R}_{\varphi} \mid \xi(x, y)=\eta(x, y)=0\right\}$ is a compact open subequivalence relation of $\mathcal{R}_{\varphi}$.

The sets $C$ and $C^{\prime}$ we will need have the following special form:

Definition 26. For $0 \leq r, r^{\prime} \leq \infty$, we define

$$
C\left(r, r^{\prime}\right)=\left\{(i, j) \in \mathbb{Z}^{2} \mid j \leq r i, j \leq r^{\prime} i\right\}
$$

with the convention $0 \cdot \infty=0$.

In addition to the notion of positive cocycle, we use the notion of small cocycle as follows.

Definition 27. Let $\theta$ be a cocycle for $(X, \varphi)$ and let $M$ be a positive integer.

Then $\theta$ is smaller or equal to $M^{-1}$ if $|\theta(X, n)| \leq 1$ for all $x \in X$ and $n=\left(n_{1}, n_{2}\right) \in \mathbb{Z}^{2}$ with $\|n\|_{\infty}=\max \left\{\left|n_{1}\right|,\left|n_{2}\right|\right\} \leq M$ and we say that $\theta$ is small if $\theta \leq \frac{1}{2}$.

If $\varphi$ is a free minimal action of $\mathbb{Z}^{2}$ on a Cantor set $X$, finding small, positive cocycles reduces to finding clopen with the following properties.

Theorem 28. Let $\varphi$ be a free minimal action of $\mathbb{Z}^{2}$ on a Cantor set $X$. Let $a, b$ be generators for $\mathbb{Z}^{2}$. Suppose that for any $N \geq 1$, there are clopen sets $A$ and $B$ such that 
1.

$$
A \cap \varphi^{-a}(B)=\varphi^{-b}(A) \cap B=\emptyset, \quad \text { 2. } A \cup \varphi^{-a}(B)=\varphi^{-b}(A) \cup B,
$$

\section{3.}

the sets $\varphi^{i(a+b)}\left(A \cup \varphi^{-a}(B)\right)$ are disjoint for $0 \leq i \leq N$.

Then for any $M \geq 1$, there exists a cocycle $\theta$ which is strictly positive on $C=\{i a+j b \mid i, j \geq 0\}$ and $\theta \leq M^{-1}$.

The preceding theorem is the result we use to find small strictly positive cocycles. for the following two classes of minimal free $\mathbb{Z}^{2}$-actions on the Cantor set.

Remark that any extension of a free minimal $\mathbb{Z}^{2}$-Cantor system with small strictly positive cocycles has the same property.

Example 29. Rotations of the group of p-adic integers.

Let $p$ be a prime number and $X=\prod_{k=0}^{\infty} \mathbb{Z} / p \mathbb{Z}$ be the abelian group of p-adic integers. If $\alpha, \beta$ are two $\mathbb{Z}$-linearly independent elements of $X$ such that either $\alpha_{0}$ or $\beta_{0}$ is non-zero, then the action $\varphi$ given for all $x \in X$ and $(i, j) \in \mathbb{Z}^{2}$ by

$$
\varphi^{(i, j)}(x)=x-i \alpha-j \beta, \quad \text { for } x \in X \text { and }(i, j) \in \mathbb{Z}^{2},
$$

is minimal and free.

As the subgroup of $X$ generated by either $\alpha$ or $\beta$ is dense, we can assume that one of the generator, $\alpha$ for example, is $(1,0,0, \cdots, 0, \cdots)$. Let $C(0, m)$ denote the cyclinder set $\left\{x \in X \mid x_{0}=x_{1}=\cdots=x_{m}=0\right\}$ and $k(m)$ the smallest positive integer such that

$$
\beta(C(0, m))=\left\{x+\beta \in X \mid x_{0}=\cdots=x_{m}=0\right\}=\alpha^{k(m)}(C(0, m)) .
$$

Then the pair of clopen sets $A=C(0, m)$ and $B=\coprod_{l=0}^{k(m)} \alpha^{l}(C(0, m))$ satisfies the first two conditions of Theorem 9.6 and therefore defines a strictly positive cocycle. A much finer construction is necessary to get a small, strictly positive cocycle.

Notice that in this example there is a short exact sequence

$$
0 \rightarrow \mathbb{Z} \rightarrow H^{1}(X, \varphi) \rightarrow \mathbb{Z}[1 / p] \rightarrow 0
$$

Example 30. Rotations of a disconnected circle.

Let $0<\alpha, \beta<\frac{1}{2}$ be two real numbers such that $\{1, \alpha, \beta\}$ is $\mathbb{Q}$-linearly independent and let us consider the action of $\mathbb{Z}^{2}$-action on the circle $\mathbb{R} / \mathbb{Z}$, by rotating by $\alpha$ and $\beta$. We then disconnect the circle along an orbit replacing each point by two separated ones and obtain a copy of the Cantor set. More precisely, if $C u t \subset \mathbb{R}$ denotes the subgroup $\{k+n \alpha+m \beta \mid k, n, m \in \mathbb{Z}\}$, we define a linear order on $\tilde{X}=\mathbb{R} \cup\left\{a^{\prime} \mid a \in C u t\right\}$ by setting $a^{\prime}<b, a<b^{\prime}, a^{\prime}<$ 
$b^{\prime}$ if $a<b$ and $a<a^{\prime}$ for all $a \in \mathbb{R}$ and consider the order topology on $\tilde{X}$. The action by translation of $\mathbb{Z}+\alpha \mathbb{Z}+\beta \mathbb{Z}$ on $\tilde{X}$ induces then a (minimal, free) action $\varphi$ of $\alpha \mathbb{Z}+\beta \mathbb{Z}$ on the Cantor set $X=\tilde{X} / \mathbb{Z}$.

For any pair of generators $a, b \in \alpha \mathbb{Z}+\beta \mathbb{Z}$, the homeomorphisms $\varphi^{a}$ and $\varphi^{b}$ are again rotations of the cut-up circle $X$. Let $N$ be a positive integer. By minimality of the rotation $R_{a} \times R_{b}$ on $\mathbb{R}^{2} / \mathbb{Z}^{2}$, there exist a positive integer $q$ and integers $i$ and $j$ such that

$$
0<q a-i<\frac{1}{2 N}, \quad 0<q b-j<\frac{1}{2 N} .
$$

Then for $0 \leq m, n<N$ and $k \in \mathbb{Z}$, we have

$$
\frac{k}{q} \leq \frac{k-i m-j n}{q}+m a+n b \leq \frac{k+1}{q} .
$$

For each $0 \leq k<q$, we can approximate $\frac{k}{q}$ by an element $x_{k} \in C u t$ such that for $0 \leq m, n<N$,

$$
x_{k}<x_{k-i m-j n}+m a+n b<x_{k+1} .
$$

Then the pair of clopen sets of $X$

$$
A=\cup_{k=0}^{q-1}\left[x_{k}, x_{k-i}+a\right), \quad B=\cup_{k=0}^{q-1}\left[x_{k}, x_{k-j}+b\right)
$$

with $k-i$ and $k-j$ interpreted modulo $q$, satisfy the assumptions of Theorem 9.6. and therefore defines a small stictly positive cocycle.

Notice that the first cohomology group of this example was computed by Forrest and Hunton in $[\mathrm{FH}]$ and is equal to $\mathbb{Z}^{3}$.

Remark 31. If $\varphi$ is a minimal $\mathbb{Z}^{2}$-action on the Cantor set, its first cohomology group $H^{1}(X, \varphi)$ always contains $\mathbb{Z}^{2}$ as a subgroup. There exists an example of such an action such that $H^{1}(X, \varphi)=\mathbb{Z}^{2}$. But this action is not free. It is not known if the first cohomology group of a free, minimal $\mathbb{Z}^{2}$-action on the Cantor set has always $\mathbb{Z}^{2}$ as a proper subgroup.

\subsection{The main results of [GPS3].}

Our main result, Theorem 32, whose proof is very long, states that if a free, minimal action $\varphi$ of $\mathbb{Z}^{2}$ on the Cantor set possesses arbitrary small, strictly positive cocycles for sufficiently many cones, then the induced étale equivalence relation $\mathcal{R}_{\varphi}$ is affable.

Theorem 32. Let $(X, \varphi)$ be a free, minimal action of $\mathbb{Z}^{2}$ on the Cantor set. Suppose that there are positive numbers $r_{\infty}, s_{\infty}$ with $s_{\infty}^{-1}-r_{\infty}^{-1} \geq 1$ satisfying the following: For every $\varepsilon>0$, there are positive real numbers $r_{\infty}+\varepsilon>r>$ $r^{\prime}>r_{\infty}$ so that for every $M \geq 1$, there is a cocycle $\theta$ on $(X, \varphi)$ such that 
1. $\theta$ is strictly positive on $C\left(r, r^{\prime}\right)$, and 2 . $\theta \leq M^{-1}$.

Similarly, for every $\varepsilon>0$, there are positive real numbers $s_{\infty}-\varepsilon<r<$ $r^{\prime}<s_{\infty}$ such that for every $M \geq 1$, there is a cocycle $\theta$ on $(X, \varphi)$ satisfying conditions 1 and 2.

Then the étale equivalence relation $\mathcal{R}_{\varphi}$ is affable.

The examples 9.7 and 9.8 described above satisfy the following stronger hypotheses.

Corollary 33. Let $(X, \varphi)$ be a free, minimal action of $\mathbb{Z}^{2}$ on the Cantor set. Suppose that for every $a, b \in \mathbb{Z}^{2}$ which generates $\mathbb{Z}^{2}$ as a group and for every $M \geq 1$, there is a cocycle $\theta$ on $(X, \varphi)$ such that

1. $\theta$ is strictly positive on $\{i a+j b \mid i, j \geq 0\}$, and 2 . $\theta \leq M^{-1}$.

Then the étale equivalence relation $\mathcal{R}_{\varphi}$ is affable.

As a consequence of Theorem 8.1,9.10, and 5.2, we then get:

Theorem 34. For $i=1,2$, let $\left(X_{i}, \mathcal{R}_{i}\right)$ be étale equivalence relations where, for each $i, X_{i}$ is totally disconnected and $\left(X_{i}, \mathcal{R}_{i}\right)$ is minimal and one of the following conditions are satisfied:

1. $\mathcal{R}_{i}$ is an AF-relation,

2. $\mathcal{R}_{i}$ arises from a free action of $\mathbb{Z}$, or

3. $\mathcal{R}_{i}$ arises from a free action of $\mathbb{Z}^{2}$ satisfying the hypotheses of 9.10.

Then the two equivalence relations are orbit equivalent if and only if there is an order isomorphism from $D_{m}\left(X_{1}, \mathcal{R}_{1}\right)$ to $D_{m}\left(X_{2}, \mathcal{R}_{2}\right)$ preserving the distinguished order units.

\section{Further developments}

Let $(X, \varphi)$ be a Cantor minimal $\mathbb{Z}^{2}$-system conjugated to the product of two Cantor minimal $\mathbb{Z}$-systems $\left(X_{1}, \varphi_{1}\right)$ and $\left(X_{2}, \varphi_{2}\right)$. By Theorem 8.1, we have that $\mathcal{R}_{\varphi_{1}}$ and $\mathcal{R}_{\varphi_{2}}$ are orbit equivalent to two AF-relations $\mathcal{R}_{1}$ and $\mathcal{R}_{2}$. As the product of two AF-equivalence relations is also $\mathrm{AF}$, we have that $\mathcal{R}_{\varphi}$ is also affable.

If $(Y, \psi)$ is an extension of the product Cantor system $\left(X_{1} \times X_{2}, \varphi_{1} \times\right.$ $\left.\varphi_{2}\right)$, it is not necessarily a product. Therefore the above argument cannot be used to show the affability of $(Y, \psi)$. In [M1], H. Matui constructs an AFsubequivalence of $\mathcal{R}_{\psi}$ satisfying the assumptions of the absorption theorem to prove the following:

Theorem 35. ([H1]). Let $\pi:(Y, \psi) \rightarrow(X, \varphi)$ be an extension between Cantor minimal $\mathbb{Z}^{2}$-systems. If $(X, \varphi)$ is conjugate to a product of two Cantor minimal $\mathbb{Z}$-systems, then $\mathcal{R}_{\psi}$ is affable. 
Recall (see for example $[\mathrm{KP}]$, p. 180) that a tiling $T$ in $\mathbb{R}^{2}$ gives rise to an an action of $\mathbb{R}^{2}$ on its continuous hull $\Omega_{T}$. If $T$ has finite local complexity and is strongly aperiodic, the hull $\Omega_{T}$ is compact and does not contain any periodic tilings. If moreover $T$ is repetitive, then the dynamical system $\left(\Omega_{T}, \mathbb{R}^{2}\right)$ is minimal.

For each tile type (or labeled tile type) $t$ in $T$, let us choose a point $x(t)$, called a puncture, in the interior of $t$. Now each tile $t \in T$ is given a puncture $x(t)$ such that if $t_{1}$ and $t_{2}$ are two tiles with $t_{2}=t_{1}+x$ for some $x \in \mathbb{R}^{2}$, then $x\left(t_{2}\right)=x\left(t_{1}\right)+x$.

The set of all the tilings $T^{\prime} \in \Omega_{T}$ such that the origin is a puncture of some tiles $t$ in $T^{\prime}$ is called the discrete hull $\Omega_{\text {punc }}$ of $T$. With the above conditions on the tiling, $\Omega_{\text {punc }}$ is a Cantor set and is a transversal to the $\mathbb{R}^{2}$-action.

An equivalence relation $\mathcal{R}_{\text {punc }}$ is defined on $\Omega_{\text {punc }}$ as follows:

$$
\mathcal{R}_{\text {punc }}=\left\{\left(T_{1}, T_{2}\right) \mid T_{i} \in \Omega_{\text {punc }} \text { and } \exists x \in \mathbb{R}^{2}: T_{1}=T_{2}+x\right\} .
$$

Then $\mathcal{R}_{\text {punc }}$ is the restriction to $\Omega_{\text {punc }}$ of the equivalence relation induced by the $\mathbb{R}^{2}$-action on the continuous hull $\Omega_{T}$.

Provided with the following topology: a sequence $\left(T_{n}, T_{n}+x_{n}\right)$ in $\mathcal{R}_{\text {punc }}$ converges to $(T, T+x)$ if and only if $T_{n} \rightarrow T$ and $x_{n} \rightarrow x, \mathcal{R}_{\text {punc }}$ is an étale equivalence relation.

In [M2], H. Matui studies the equivalence relation $\mathcal{R}_{\text {punc }}$ associated to a substitution tiling. Recall that a substitution tiling system in $\mathbb{R}^{2}$ consists of a pair $(\mathcal{V}, \omega)$ where $\mathcal{V}$ is a finite collection of polygons in $\mathbb{R}^{2}$, the prototiles, and $\omega$ is a substitution rule. We also have an inflation constant $\lambda>1$ such that for every $p \in \mathcal{V}, w(p)$ is a finite collection of tiles (a tile is a translate of one prototile) with pairwise disjoint interiors and their union is $\lambda p=\{\lambda v \mid v \in p\}$. The Penrose tiling is an example of a substitution tiling.

For a substitution tiling system which is primitive, aperiodic and satisfies the finite pattern condition, I. Putnam constructs in [P1] a minimal AF subequivalence relation $\mathcal{R}$ of $\mathcal{R}_{\text {punc }}$. The equivalence relation $\mathcal{R}$ is too large to apply the Absorption Theorem 7.1. In [H2], Matui constructs a smaller AF-subequivalence relation $\mathcal{R}^{\prime} \subset \mathcal{R}$ satisfying the conditions of Theorem 7.1 and obtains:

Theorem 36. ([H2]). Let $(\mathcal{V}, \omega)$ be a substitution tiling system in $\mathbb{R}^{2}$ as above. Then the equivalence relation $\mathcal{R}_{\text {punc }}$ on $\Omega_{\text {punc }}$ is affable.

In a work in progress, Giordano, Matui, Putnam and Skau have generalized the Absorption Theorem presented in Section 7. With this gneralization, the first step of the strategy presented in Section 6 is now easier to implement. In particular for a minimal free action of $\mathbb{Z}^{2}$ on the Cantor set, we are now able to construct a minimal AF-subequivalence relation of $\mathcal{R}_{\varphi}$ satisfying the assumptions of the new absorption theorem without having to use cocycles. Theorem 9.12 can therefore be extended to cover all free minimal action of $\mathbb{Z}^{2}$ on the Cantor set. 


\section{References}

[BBG] J. Bellissard, R. Benedetti and J.-M. Gambaudo, Spaces of Tilings, Finite Telescopic Approximations and Gap-Labelling, preprint.

[BG] R. Benedetti and J.-M. Gambaudo, On the dynamics of G-solenoids: application to Delone sets, Erg. Th. and Dyn. Sys.,23, (2003), p. 673-691.

[CFW] A. Connes, J. Feldman and B. Weiss, An amenable equivalence relation is generated by a simple transformation. J. Erg. Th. and Dyn. Sys.,1, (1981), p. 431-450.

[DJK] R. Dougherty, S. Jackson and A.S. Kechris, The structure of hyperfinite Borel equivalence relations, Trans. Amer. Math. Soc., 341, (1994), p. 193-225.

[D1] H. Dye, On groups of measure preserving transformations I, American J. Math, 81, (1959), 119-159.

[D2] H. Dye, On groups of measure preserving transformations II, American J. Math, 85, (1963), 551-576.

[Ef] E.G. Effros, Dimensions and $C^{*}$-algebras, Conf. Board Math. Sci., 46, Amer. Math. Soc., Providence, R.I., 1981.

[FM] J. Feldman and C.C. Moore, Ergodic equivalence relations, cohomology and von Neumann algebras I, Trans. Amer. Math. Soc., 234, (1977), p. 289-324.

[F] A. Forrest, A Bratteli diagram for commuting homeomorphism of the Cantor set, Internat. J. Math. 11 (2000), p. 177-200.

$[\mathrm{FH}]$ A. Forrest and J. Hunton The cohomology and K-theory of commuting homeomorphisms of the Cantor set. Erg. Th. and Dyn. Sys.,19,(1999), 611-625.

[GPS1] T. Giordano, I.F. Putnam and C.F. Skau, Topological orbit equivalence and $C^{*}$-crossed products, J. reine angew. Math. 469 (1995), p. 51-111.

[GPS2] T. Giordano, I.F. Putnam and C.F. Skau, Affable equivalence relations and Cantor minimal, Ergod. Theor. and Dynam. Syst., 23, (2004), p. 441-475.

[GPS3] T. Giordano, I.F. Putnam and C.F. Skau, The Orbit Structure of Cantor Minimal $\mathbb{Z}^{2}$-systems, preprint.

[HPS] R.H. Herman, I.F. Putnam and C.F. Skau, Ordered Bratteli diagrams, dimension groups, and topological dynamics, Internat. J. Math. 3 (1992), p. $827-864$.

[HM] G. Hjorth and M. Molberg, Free continuous actions on zero dimensional spaces, to appear in Topology and its applications.

[KP] J. Kellendonk and I.F. Putnam, Tilings, $C^{*}$-algebras, and K-theory, Directions in Mathematical Quasicrystals, 117-206, CRM Monogr. Ser. 13, Amer. Math. Soc., Providence, RI, 2000.

[Kr] W. Krieger, On a dimension for a class of homeomorphism groups, Math. Ann., 252, (1980), p. 87-95.

[K] K. Kuratowski, Topology, Vol II, Academic Press, New-York 1968.

[M1] H. Matui, A short proof of affability for certain Cantor minimal $\mathbb{Z}^{2}$-systems, preprint.

[M2] H. Matui, Affability of equivalence relations arising from two-dimensional substitution tilings, preprint.

[OW] D.S. Ornstein and B. Weiss, Ergodic theory of amenable group actions I: The Rohlin lemma, Bull. Amer. Math. Soc., 2, (1980), p. 161-164.

[Pa] A. L. T. Paterson, Groupoids, Inverse Semigroups and their Operator Algebras. (Progress in Mathematics, vol. 170), Birkhäuser, Boston-Basel-Berlin, 1999.

[Ph] N.C. Phillips, Crossed product of the Cantor set by a free, minimal action of $\mathbb{Z}^{n}$, Comm. Math. Phys., to appear. 
[P] I.F. Putnam, The $C^{*}$-algebras associated with minimal homeomorphisms of the Cantor set, Pacific J. Math.., 136, (1989), p. 329-353.

[P1] I.F. Putnam, The ordered K-theory of $C^{*}$-algebras associated with substitution tilings, Comm. Math. Phys., 214, (2000), p. 593-605.

[R] J. Renault, A groupoid approach to $C^{*}$-algebras. Lect. Notes Math. 739, Springer-Verlag, 1980.

[R1] J. Renault, AF-equivalence relations and their cocycles. Preprint (2001).

[W] B. Weiss, Private communications. 